\title{
Biruh Tesfa procure aux employées domestiques, aux orphelines et aux immigrantes des zones urbaines éthiopiennes une assistance sociale, une formation sur le VIH ainsi qu'un programme de développement des compétences
}

Annabel Erulkar

Population Council

Belaynesh Semunegus

Population Council

Gebeyehu Mekonnen

Population Council

Follow this and additional works at: https://knowledgecommons.popcouncil.org/departments_sbsr-pgy

Part of the Demography, Population, and Ecology Commons, Family, Life Course, and Society

Commons, Gender and Sexuality Commons, and the International Public Health Commons

How does access to this work benefit you? Let us know!

\section{Recommended Citation}

Erulkar, Annabel, Belaynesh Semunegus, and Gebeyehu Mekonnen. 2011. "Biruh Tesfa procure aux employées domestiques, aux orphelines et aux immigrantes des zones urbaines éthiopiennes une assistance sociale, une formation sur le VIH ainsi qu'un programme de développement des compétences," Promouvoir la santé, la sécurité et la productivité transitions vers l'âge adulte Bulletin d'information no. 21. New York: Population Council. 


\section{Biruh Tesfa procure aux employées domestiques, aux orphelines et aux immigrantes des zones urbaines éthiopiennes une assistance sociale, une formation sur le VIH ainsi qu'un programme de développement des compétences}

\section{Rédigé par Annabel Erulkar, Belaynesh Semunegus et Gebeyehu Mekonnen}

E n Éthiopie, l'épidémie de VIH se révèle de plus en plus importante en milieu urbain et chez la population féminine. Selon les estimations, 7,7 pourcent de la population urbaine éthiopienne est séropositive, par rapport à moins d'un pourcent de la population rurale (MOH 2007). Ces chiffres suggèrent une épidémie urbaine généralisée et une épidémie rurale concentrée parmi les groupes à risque (Myrick et Cassell 2007). À l'échelle nationale, le rapport femme/homme en termes d'infection par le VIH est de 3 pour 2, traduisant ainsi la vulnérabilité accrue des jeunes filles et des femmes face au VIH. II est possible que les jeunes filles qui migrent depuis les zones rurales vers les milieux urbains soient moins protégées, plus vulnérables face à la violence et aux agressions sexuelles, et également moins bien équipées pour se prémunir contre ces situations d'abus (toutes susceptibles d'accroître le risque d'infection par le VIH).

Une étude menée dans les zones à faibles revenus d'Addis-Abeba a montré que 45 pourcent des adolescentes vivant dans ces zones étaient immigrantes. Parmi ces immigrantes, 87 pourcent exerçaient des emplois précaires, notamment des travaux domestiques, caractérisés par de mauvaises conditions de travail et de faibles rémunérations et occupés principalement par des jeunes filles et des femmes (UNICEF 1999). Les employées domestiques ayant participé à l'étude menée par le Population Council à Addis-Abeba ont indiqué avoir travaillé en moyenne 64 heures pendant la semaine précédent l'enquête, avoir touché un salaire mensuel moyen de 52 Birr, soit environ 6 \$ US (Erulkar et Mekbib 2007). Une étude plus récente réalisée par le Council auprès d'adolescentes dans les zones urbaines insalubres d'Éthiopie a permis de constater que les employées domestiques sont davantage exposées au risque d'agressions sexuelles, y compris de rapports sexuels non consensuels (Erulkar et Ferede 2009). En outre, les heures de travail prolongées et l'isolement social des employées domestiques empêchent un grand nombre d'entre elles de bénéficier des programmes d'intégration des jeunes (Erulkar et al. 2006).

\section{Le programme Biruh Tesfa}

Biruh Tesfa (terme amharique qui signifie «Avenir radieux») a pour objet de résoudre le problème de l'isolement social des adolescentes déscolarisées âgées de 10 à 19 ans, y compris les migrantes des

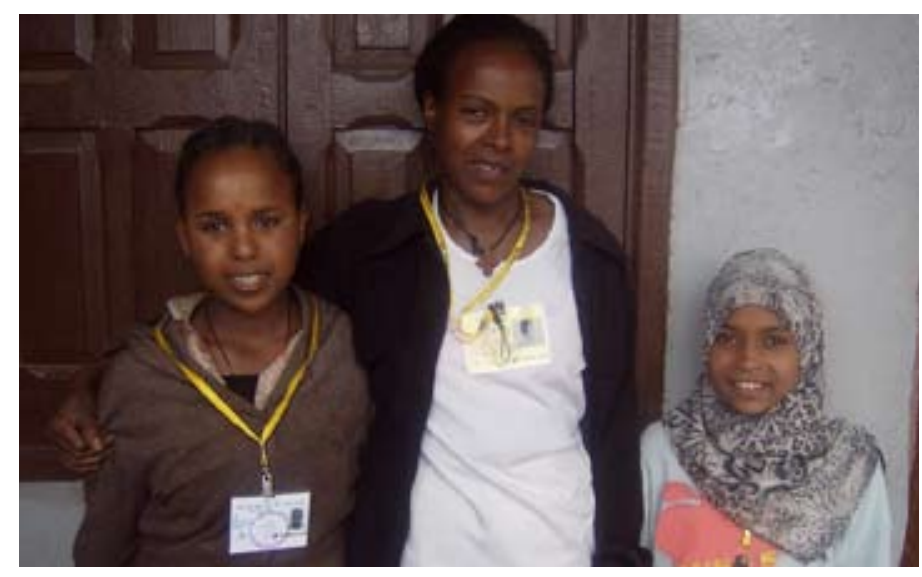

zones rurales vers les zones urbaines, les employées domestiques et les orphelines. À l'aide d'un modèle de tutorat collectif, les jeunes filles développent leur capital social et accèdent à des programmes d'alphabétisation, d'acquisition de compétences et d'information sur le $\mathrm{VIH}$, la santé génésique et la violence dirigée contre les femmes. Ce projet est mis en œuvre par le Bureau de la jeunesse et des sports dans les régions d'Addis-Abeba et d'Amhara ainsi que dans les administrations qebelé d'Addis-Abeba, Baher Dar, Debre Markos, Dessie et Gondar. Le Population Council fournit une assistance technique et a dirigé l'évaluation du projet pilote dans le quartier Mercado d'Addis-Abeba.

\section{Recrutement en porte-à-porte pour entrer en contact avec les} populations les plus marginalisées et invisibles Le programme Biruh Tesfa réunit les jeunes filles déscolarisées en groupes amenés à rencontrer des tutrices adultes dans des espaces collectifs. Ces tutrices et superviseurs sont recrutés par l'administration de qebelé et les responsables locaux. Les tutrices sont âgées de 20 à 40 ans, possèdent une formation scolaire d'au moins 12 ans, résident sur le site du projet et sont des membres connus et respectés de la communauté. Les tutrices sont formées pendant une semaine par l'entremise d'un programme centré sur les aptitudes personnelles et sociales, le VIH/SIDA, la santé génésique, les questions d'égalité 
des sexes - y compris la violence envers les femmes - ainsi que l'alphabétisation élémentaire.

À l'issue de cette formation, les tutrices identifient méthodiquement les jeunes filles déscolarisées remplissant les conditions de participation au programme. Les tutrices se rendent dans chaque maison et établissent une liste informelle des membres du foyer, y compris leur sexe, leur âge et leur statut scolaire. Les tutrices interrogent également les membres du foyer sans lien familial, y compris les employées domestiques.

Les jeunes filles remplissant les conditions requises (déscolarisées, âgées de 10 à 19 ans) sont invitées à participer au programme. Les tutrices s'assurent également d'obtenir la permission des parents, des tuteurs ou - dans le cas d'employées domestiques - des employeurs, qui sont rarement favorables à leur participation au premier abord. Les tutrices sont ensuite chargées de suivre les jeunes filles qu'elles inscrivent au programme. En cas d'absence d'une jeune fille à trois réunions de groupe ou plus, les tutrices se rendent au domicile de la jeune fille afin de connaître le motif de son absence. Souvent, l'employeur de la jeune fille a refusé de la laisser participer au programme, auquel cas les tutrices tentent de renégocier au nom de la jeune fille.

Lorsque les tutrices prenaient les inscriptions en faisant du porteà-porte, je me suis inscrite. Lorsque j'en ai entendu parler pour la première fois, je ne croyais pas que cela se passerait réellement. Je pensais que je ne pourrais pas y participer car mes employeurs sont très stricts et ne m'autorisent pas à sortir. [D'abord,] mon employeur ne voulait pas me laisser y assister. Elle disait qu'il n'était pas bon de laisser sortir les domestiques car lorsqu'elles se rencontreraient, elles discuteraient entre elles de leurs salaires et demanderaient ensuite plus d'argent. Elles seraient alors trop gâtées et ne travailleraient plus aussi durement. Ma tutrice nous a rendu visite et m'a demandé pourquoi j'avais été absente, et je le lui ai expliqué.... Elle en a parlé avec mon employeur et a fini par la convaincre. Mais je ne peux pas encore venir de façon régulière car j'ai trop de travail à faire.

\section{- Fille de Bahir Dar, 19 ans, orpheline de mère et de père,} n'a jamais fréquenté l'école, employée domestique

\section{Au-delà de l'information et des compétences : une identité et des attaches}

Les groupes de jeunes filles se réunissent dans les salles et les établissements de la collectivité mis à disposition par l'administration locale à une heure qui leur convient. Les réunions ont généralement lieu trois à cinq fois par semaine. Les groupes sont formés en fonction des âges et des niveaux scolaires, y compris les jeunes filles qui ne sont jamais allées à l'école, celles qui possèdent quelques aptitudes de lecture et d'écriture et celles qui maîtrisent la lecture. Les programmes d'alphabétisation et d'aptitude au calcul sont adaptés au niveau de chaque groupe, et tous les groupes reçoivent une formation dans le domaine des aptitudes personnelles et sociales, du VIH/SIDA et de la santé génésique. Toutes les jeunes filles participant au programme reçoivent une carte d'identité du projet avec leur photo. Pour nombre d'entre elles, cette simple carte d'identité est la seule forme d'identification qu'elles possèdent et bien souvent la seule photo d'elles-mêmes dont elles disposent. Quatre cahiers d'exercice par an ainsi que des stylos et des crayons leur sont procurés. En raison des conditions de pauvreté extrême qui sont les leurs, la plupart des bénéficiaires ne disposent même pas des biens de première nécessité, tels que du savon ou des serviettes hygiéniques. Chaque année, environ quatre pains de savon et des serviettes hygiéniques produites localement sont fournis à ces jeunes filles. Au total, chaque jeune fille reçoit l'équivalent de 95 Birr/7,75 \$ US en aide matérielle par an. Si elles semblent insignifiantes, ces ressources sont difficiles d'accès pour les bénéficiaires du programme Biruh Tesfa (Erulkar et al. 2008).

\section{Fournir un accès aux services fondamentaux}

Une grande partie des participantes arrivant de zones rurales reculées, il est possible qu'elles ne se soient jamais rendues dans un établissement médical. Les bénéficiaires du projet font l'objet d'examens médicaux réalisés dans des cliniques publiques locales afin de contrôler leur état de santé. Les jeunes filles nécessitant des soins curatifs élémentaires sont prises en charge gratuitement moyennant un accord conclu avec les établissements de santé publics. En outre, le Council développe actuellement un réseau d'établissements d'aiguillage dans les secteurs public et privé sur chaque site du projet. Ces établissements fourniront des services subventionnés ou gratuits par l'entremise d'un système d'aiguillage par coupons distribués par les tutrices aux jeunes filles nécessitant des soins.

Loin de leurs proches ou d'autres structures de protection, les employées domestiques et autres migrantes sont vulnérables face à la menace de l'exploitation et des agressions sexuelles. Les employées domestiques sont particulières exposées dans la mesure où elles dépendent souvent de leurs employeurs pour subvenir à leurs besoins fondamentaux et se loger. Les victimes d'exploitation et d'abus sexuels ne disposent généralement d'aucune source d'aide ou de soutien. Le programme Biruh Tesfa a fondé un partenariat avec l'Organization for the Prevention, Rehabilitation and Integration of Female Street Children (OPRIFS - Organisation pour la prévention, la réinsertion et l'intégration des jeunes filles de la rue) qui accueille et soutient les jeunes filles victimes de violences sexuelles. Grâce à ses refuges d'Addis-Abeba et de Bahir Dar, I'OPRIFS offre un logement aux jeunes filles victimes d'actes de violence, fréquemment perpétrés par un employeur ou un membre de la famille. Les victimes bénéficient de conseils, d'une assistance juridique et se lient d'amitié avec les autres filles du refuge. Les conseillers de l'OPRIFS rendent également visite aux groupes du programme Biruh Tesfa pour enseigner aux jeunes filles comment réduire les risques auxquelles elles s'exposent, et pour faire connaître ses services.

Ouvrir la voie vers des moyens de subsistance sécurisés et productifs En raison de leurs faibles niveaux scolaires et d'un accès restreint aux réseaux d'assistance, les employées domestiques rencontrent des difficultés pour finaliser la transition vers des formes de travail plus sûres et plus gratifiantes. En partenariat avec la Fondation Nia, chaque année 40 bénéficiaires du programme Biruh Tesfa, principalement des employées domestiques et des orphelines, reçoivent une formation de développement de leurs compétences dans le secteur de la beauté (coiffure, soins de la peau et onglerie). Les jeunes filles désireuses de quitter leur emploi domestique et de bénéficier d'une formation professionnalisante vivent au 
domicile de leurs employeurs. Aussi, les bénéficiaires du programme Nia se voient proposer un logement à proximité du centre de formation ainsi qu'un emploi à temps partiel qui leur permet de gagner de l'argent pour subvenir à leurs besoins fondamentaux. Une fois la formation validée, les jeunes diplômées sont placées dans des salons de sorte qu'elles puissent commencer immédiatement à gagner de l'argent et ne soient pas contraintes de réintégrer leur emploi domestique.

\section{Accroître l'implication des jeunes filles handicapées}

Lors des premières phases du programme, les tutrices ont pu déterminer qu'il était aussi difficile de recruter des jeunes filles handicapées que des employées domestiques. Certains parents ou tuteurs n'élèvent pas leurs enfants handicapés de manière à ce qu'ils puissent mener une vie indépendante et se montrent réticents à l'idée de les laisser quitter leur environnement familial protégé. En outre, une fois recrutées, de nombreuses jeunes filles handicapées se heurtent à des problèmes logistiques tels que le transport ou l'accompagnement sur le site du programme.

Par conséquent, le programme Biruh Tesfa a fondé un partenariat avec l'Ethiopian Women with Disabilities National Association (EWDNA -

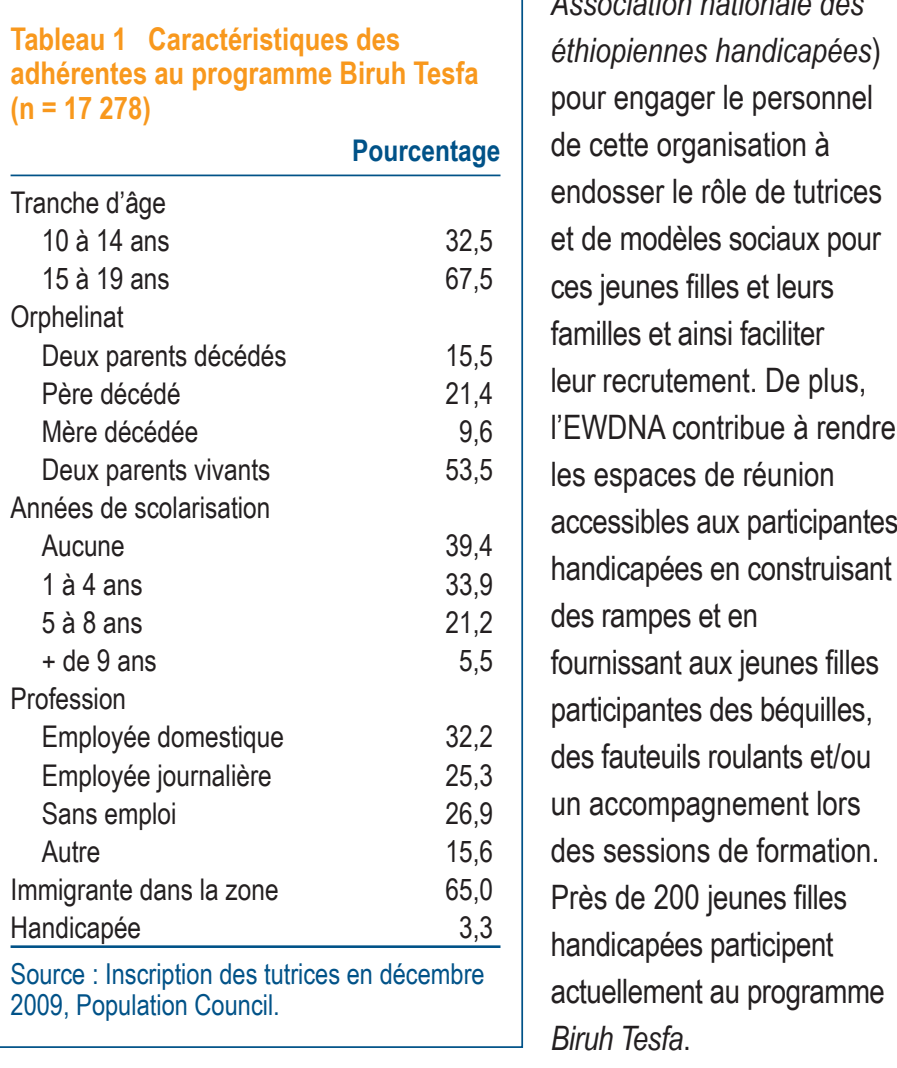

Agir en faveur des jeunes filles les plus vulnérables d'Éthiopie En décembre 2009, plus de 17000 jeunes filles avaient bénéficié du programme Biruh Tesfa à Addis-Abeba, Bahir Dar, Debre Markos, Dessie et Gondar. Les participantes sont extrêmement vulnérables : près de la moitié (47 pourcent) d'entre elles ont perdu un ou deux parents ; près de 40 pourcent d'entre elles n'ont reçu aucune éducation et 73 pourcent d'entre elles enregistrent un cursus scolaire inférieur à cinq ans. Trente deux pourcent d'entre elles sont des employées domestiques et 25 pourcent sont des employées journalières.
Je me suis enfuie pour me réfugier à Bahir Dar lorsque mon père a tenté de me marier contre mon gré... Je suis donc allée voir une dame que je connaissais et lui ai demandé de me conduire à Bahir Dar. Quand je vivais en zone rurale, j'ai entendu parler d'une ville appelée Bahir Dar où personne n'était contraint d'aller chercher de l'eau ou du bois et où la vie était facile. Elle m'a donc accompagnée jusqu'au domicile de sa fille [à Bahir Dar] pour m'y placer comme employée domestique et elle a négocié un salaire de 12 Birr [1 \$ US] par mois. Même si nous nous étions mis d'accord pour 12 Birr, elle ne m'a jamais payée. Parfois, elle me donnait des vêtements.

\section{_Fille de Bahir, âgée de 19 ans, employée domestique,} scolarisée pendant trois ans

Je suis née dans la zone rurale de Gojjam (région d'Amhara)... Ma mère est morte quand j'avais huit ans et trois ans plus tard, mon père est décédé.... Ma grand-mère s'est alors occupée de moi, mais elle est morte également deux ans plus tard.... Ma tante, qui vit à AddisAbeba m'a amenée ici et a promis de m'envoyer à l'école. Dès mon arrivée, elle m'a enfermée dans sa maison et ne m'a plus laissée sortir. Je devais aider aux tâches ménagères. Lorsque je suis arrivée ici, j'étais en bonne santé, mes jambes fonctionnaient normalement et je pouvais marcher. Un jour, alors que j'étais en train de travailler, je suis tombée et je me suis cassé les deux jambes. Je ne suis pas allée à l'hôpital et je suis devenue handicapée. Je suis restée à la maison avec ma tante et elle m'insultait, me disait que je ne «valais rien» et que j'étais «inutile»... Aujourd'hui, je vis dans la rue. Je vis de ce que je parviens à vendre dans la rue. J'ai commencé à vendre des bonbons et du chewing gum...

\section{_Fille d'Addis-Abeba, 16 ans, orpheline de père et de mère, vit dans la rue, vendeuse de rue, scolarisée pendant un an}

L'évaluation du programme Biruh Tesfa indique un succès rapide Le Population Council a évalué le programme Biruh Tesfa sur son site pilote dans le quartier de Mercato à Addis-Abeba. Avant sa mise en œuvre, des enquêtes ont été menées auprès de jeunes filles âgées de 10 à 19 ans dans des communautés expérimentales et témoins à Addis-Abeba, ainsi que des enquêtes de suivi deux ans plus tard. Ces enquêtes ont permis d'évaluer les changements apportés à l'échelle de la communauté à la suite du projet, avec un intérêt particulier porté à la participation sociale et aux systèmes de protection sociale. Les statistiques de service ont permis de suivre la participation et d'établir le profil des bénéficiaires.

En situation de référence, 29 pourcent des jeunes filles sur le site expérimental ont indiqué avoir «de nombreux amis», tandis qu'en fin d'expérience, 35 pourcent indiquaient avoir beaucoup d'amis. Sur le site témoin, aucune évolution du pourcentage de jeunes filles indiquant avoir de nombreux amis n'a été observée (21 pourcent en référence comme en fin d'expérience). En situation de référence, seulement 7 pourcent des jeunes filles remplissant les conditions de participation au programme dans la zone de Mercato ont indiqué disposer d'un «espace sécurisé» dans leur communauté pour rencontrer leurs amies. En fin d'expérience, 25 pourcent des jeunes filles ont indiqué disposer d'un espace sécurisé, soit un écart statistique considérable. 
Figure 1 Proportion de filles indiquant avoir beaucoup d'amis dans leur quartier, en situation de référence et en fin d'expérience par site

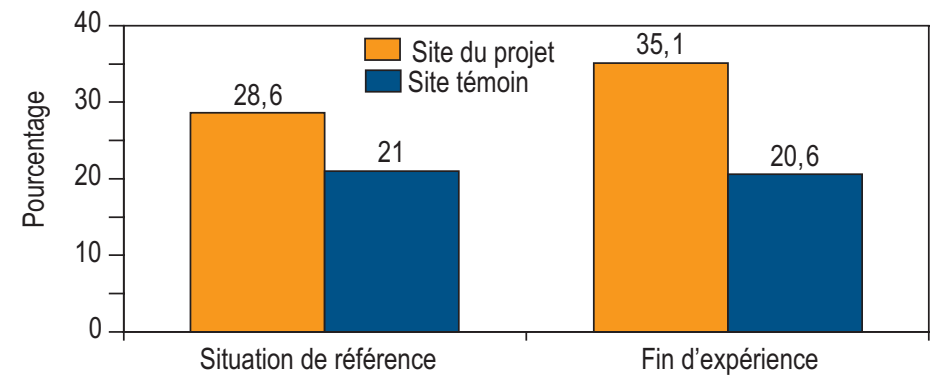

Remarque : Analyse préliminaire du Population Council en fonction de données pondérées.

Cette évaluation comprenait une variable composite traduisant une participation sociale, appréciée par le biais des réseaux relationnels, des liens nouvellement noués ou de la participation aux groupes de jeunes filles. En fin d'expérience, il était bien plus probable que les jeunes filles sur le site expérimental soient considérées comme «socialement participatives» par rapport aux jeunes filles du site témoin. En situation de référence, il était considérablement moins probable que les jeunes filles sur le site du projet indiquent disposer de systèmes de protection sociale, comme par exemple un proche susceptible de leur prêter de l'argent ou un autre endroit où se loger. En fin d'expérience, aucune différence n'a été constatée en matière de systèmes de protection sociale entre les jeunes filles des deux sites, suggérant par là que le programme Biruh Tesfa a permis d'améliorer la condition des jeunes filles de cette zone. En fin d'expérience, il était bien plus probable que les jeunes filles sur le site du projet aient bénéficié de conseils bénévoles et d'un dépistage du VIH, par rapport aux jeunes filles du site témoin.

Les entretiens avec les jeunes filles ont mis en relief les changements apportés dans leurs vies grâce au programme Biruh Tesfa.

Mes parents sont morts quand j'étais enfant.... Je ne me souviens pas bien de cette époque. J'ai vécu avec ma tante jusqu'à l'âge de 12 ans, puis je suis venue ici pour vivre avec mon oncle et aller à l'école. Un autre membre de la famille vivait également avec mon oncle. Un jour, cet homme m'a violée alors que j'étais seule à la maison, et je suis tombée enceinte. Lorsque mon oncle a découvert que j'étais enceinte, il m'a chassée de la maison. Puis, une dame m'a recueillie ; mon enfant et moi vivons actuellement avec elle ; je l'aide pour les tâches ménagères.... Depuis que j'ai commencé à suivre ce programme, j'ai appris comment me protéger contre la violence et que faire si l'on me persécute. Je pense que si j'avais pu participer à ce programme plus tôt, je n'aurais peut-être pas été violée par cette personne et je ne serais pas tombée enceinte....

-Fille de Gondar, 15 ans, orpheline de mère et de père, n’a jamais fréquenté l'école, employée domestique
Je suis venue ici [suivre le programme] avec intérêt et détermination. J'ai pu gagner en assurance et découvrir que je pouvais faire face à mes problèmes et les résoudre. Je pense que, quoi qu'il arrive, si je travaille dur, j'y arriverai.

_Fille d'Addis-Abeba, 16 ans, orpheline de père et de mère, vient de la région d'Amhara, scolarisée pendant un an

Biruh Tesfa s'étend désormais à 12 villes supplémentaires en Éthiopie. En outre, au regard de la demande de participation au programme exprimée par des filles plus jeunes, les tutrices s'attachent actuellement à créer des groupes destinés aux jeunes filles âgées de 7 à 9 ans.

\section{Bibliographie et ressources}

Erulkar A et Ferede A. 2009. "Social exclusion and early or unwanted sexual initiation among poor urban females in Ethiopia." International Perspectives on Sexual and Reproductive Health, 35(4): 186-193.

Erulkar A, Ab Mekbib T, Tegegne M. 2008. Biruh Tesfa: "Creating a 'Bright Future' for migrant girls in urban areas of Ethiopia." Population Council Transitions to Adulthood Brief series no.21. New York: Population Council.

Erulkar A, Mekbib T. 2007. "Invisible and vulnerable: Adolescent domestic workers in Addis Ababa, Ethiopia." Vulnerable Children and Youth Studies, December; 2(3): 246-256.

Erulkar A, Mekbib T, Simie N, Gulema J. 2006. "Differential use of adolescent reproductive health programs in Addis Ababa, Ethiopia." Journal of Adolescent Health, 38, 253-260.

Erulkar A, Semunegus B, Mekonnen G . 2010. "Biruh Tesfa ('Bright Future') Program Provides Domestic Workers, Orphans and Migrants in Urban Ethiopia with Social Support, HIV Education and Skills." Population Council Ethiopia Program Brief Series.

Human Rights Watch (HRW). 2006. "Swept under the rug: Abuses against domestic workers around the world." Human Rights Watch, 18.

Ministry of Health (MOH) and Federal HAPCO. 2007. "Single Point HIV Prevalence Estimate," Addis-Abeba, Éthiopie.

Myrick R, Cassell M. 2007. "Meeting the prevention needs of high-risk populations in Ethiopia," presentation at CDC-Eth, 26 avril 2007.

UNICEF. 1999. Child Domestic Work. Innocenti Digest. Florence: UNICEF.

\section{Bailleurs de fonds}

UNFPA, USAID/PEPFAR, Nike Foundation, United Nations Foundation, DFID, Fisher Family Foundation ainsi que des particuliers.

\section{Institutions partenaires}

Ministère éthiopien de la jeunesse et des sports ; Bureau de la jeunesse et des sports à Addis-Abeba ; Bureau de la jeunesse et des sports de la région d'Amhara; Administrations qebelé d'Addis-Abéba, Bahir Dar, Debre Markos, Dessie et Gondar ; Ethiopian Women with Disabilities National Association (EWDNA) ; Fondation $\mathrm{Nia}$; Organization for the Prevention, Rehabilitation and Integration of Female Street Children (OPRIFS) ; Young Women's Christian Association (YWCA)

\section{P Population Council}

Le Population Council fait évoluer les perspectives sur les questions essentielles de santé et de développement. Nous cherchons à comprendre les causes et les conséquences de l'inégalité entre les sexes et des disparités sociales qui s'installent au cours de l'adolescence. Nous fournissons des données en vue d'améliorer les programmes et les politiques mis en place sur le terrain qui permettent d'assurer des transitions efficaces et productives vers la vie adulte dans les pays en développement. www.popcouncil.org

(c) 2011 The Population Council, Inc.

4 - Rendez-vous sur www.popcouncil.org/publications/serialsbriefs/TABriefs.asp pour consulter tous les bulletins d'information de la série Promoting healthy, safe, and productive transitions to adulthood [Promouvoir des transitions saines, sécurisées et productives vers l'âge adulte]. 